\title{
Evaluating the Effect of Vitamin A on Persistent Wheezing in Infants With Bronchiolitis in Southern Iran in 2018
}

\author{
Gholamreza Soleimani (iD ${ }^{1}$, Elham Shafighi Shahri (iD ${ }^{2}$, Alireza Ganjali (iD ${ }^{3}$, Alireza Ansari Moghadam \\ (iD) ${ }^{3}$ and Afsaneh Mirshekari ${ }^{2,{ }^{*}}$ \\ ${ }^{1}$ Department of Pediatric, Research Center for Children and Adolescents, Zahedan University of Medical Sciences, Zahedan, Iran \\ ${ }^{2}$ Departments of Medicine, Zahedan University of Medical Sciences, Zahedan, Iran \\ ${ }^{3}$ Department of Epidemology and Biostatistics, Health Promotion Research Center, Zahedan University of Medical Sciences, Zahedan, Iran \\ "Corresponding author: Departments of Medicine, Zahedan University of Medical Sciences, Zahedan, Iran. Tel: +98-9155422991. Email: afsanemir4@gmail.com
}

Received 2021 June 15; Revised 2021 October 18; Accepted 2021 October 23.

\begin{abstract}
Background: Bronchiolitis is a viral infection that causes the airways (bronchioles) in the lungs to become narrow, which makes breathing difficult.

Objectives: This study aimed to evaluate the effect of vitamin A on persistent wheezing in infants with bronchiolitis.

Methods: This study was performed on 60 infants with bronchiolitis who were randomly divided into two equal groups ( $\mathrm{n}=30$ ) of intervention (receiving 5000 units per $\mathrm{kg}$ of vitamin A injection) and control. The severity of wheezing and retraction and number of breaths in the patients were recorded. Chi-square, Mann-Whitney U test, and t-test were used to compare the data.

Results: Our study showed that $6.7 \%$ of infants in the control group had severe wheezing during the discharge, while no wheezing was reported in the intervention group. Also, the rate of moderate wheezing in the control and intervention groups was $40 \%$ and $10 \%$, respectively $(\mathrm{P}=0.006)$. Two weeks after treatment, the moderate wheezing frequency in the control group was $30 \%$, but no wheezing was recorded in the intervention group, which was statistically significant $(\mathrm{P}<0.001)$.

Conclusions: In this study, administration of vitamin A significantly reduced the severity of wheezing in patients with bronchiolitis. Accordingly, it is recommended that vitamin A along with other treatments, be prescribed for all infants with symptoms of bronchiolitis.
\end{abstract}

Keywords: Bronchiolitis, Wheezing, Vitamin A

\section{Background}

Bronchiolitis is a viral infection that causes the airways (bronchioles) in the lungs to become narrow, which makes breathing difficult. It occurs most often in children under two years of age during winter and early spring. Very rarely, adults can also get bronchiolitis (1). In fact, it is the most common infection of the lower respiratory tract in infants and the main reason for admission from birth to 14 months of life (2). It is also one of the most important causes of mortality and morbidity in developing countries (3). This disease is the most common infection in the lower airways during the first year of life, and approximately one percent of children are hospitalized annually (4). The disease is self-limiting and is mainly associated with respiratory syncytial virus (RSV), and bronchial obstruction is associated with edema and accumulation of mucus (5). Generally, bronchiolitis is established by a syncytial pattern (6). The prevalence is $2: 1$ in males (7), and the highest inci- dence is in winter and more by RSV, although other viruses are also involved., although other viruses are also involved (6). Bronchiolitis is commonly defined as the first viral respiratory distress attack with symptoms of coryza, coughing, crackle, and wheezing, but its clinical definition varies from country to country $(8,9)$. The American Academy of Pediatrics (AAP) has defined bronchiolitis as a set of clinical signs and symptoms, including early symptoms of respiratory infections and wheezing in children younger than two years of age (8). However, in the UK and some parts of Europe and Australia, wheezing is not a mandatory criterion for diagnosis $(8,9)$. Usually, the first sign of the disease in infants is rhinorrhea. Coughing can occur simultaneously, but it usually occurs after one to three days, which can be accompanied by mild cough and sneezing at this time. Immediately after coughing, a child with bronchiolitis will have audible wheezing. If the disease is mild, the symptoms do not progress more than this stage. Auscultation often represents mild and diffused dry crackles with 
exhalation wheezing (10). In about half of the cases, the disease continues with a recurrent wheezing after bronchiolitis, which is referred to as wheezing after bronchiolitis. Some studies investigated the association of this wheezing with asthma, which indicated the potential role of inflammatory and interleukin processes in the onset of the disease and its related symptoms (5, 10-12). Sustained wheezing that develops after bronchiolitis as a respiratory tract disease is one of the causes of morbidity in children, besides being costly for the health system $(13,14)$.

According to recent studies, antioxidants (such as vitamins A, E, and C, and carotenoids) have a unique effect on the immune system (15). Vitamin A has important roles such as regulating the functions of immunity, vision, reproduction, and cellular communication. Moreover, different studies have shown its role in the health of children under the age of five, so that even a mild deficiency of this vitamin leads to increased mortality in children aged six months to six years (16-20). Regarding the proven role of vitamin A in regulating immune function, (21) some studies have examined the relationship between this vitamin and infant bronchiolitis.

Considering the relationship between bronchiolitis and wheezing and asthma in infants (11) and due to the limited studies on the efficacy of administrating vitamin A for patients with bronchiolitis, this study was carried out to determine the effect of vitamin A on persistent wheezing in infants with bronchiolitis referred to Ali Ibn Abi Talib Hospital in southern Iran in 2018.

\section{Objectives}

The present study aimed to determine the effect of vitamin A on persistent wheezing in infants with bronchiolitis referred to Ali Ibn Abi Taleb Hospital in Zahedan, Iran.

\section{Methods}

This clinical trial was conducted on infants (age range: one month to two years of age) diagnosed with bronchiolitis after taking a history and performing a physical examination. The exclusion criteria were a history of congenital heart disease, pulmonary disease, asthma, immune deficiency, and other chronic underlying diseases. Patients were also excluded from the study if another diagnosis was made during the diagnostic or treatment process.

The patients were randomly divided into two groups of case and control. The patients' checklists were completed considering their demographic information (such as age and sex) and severity of symptoms (wheezing severity, retraction status, respiratory rate, and general condition of the patient). Both groups received the usual treatments for bronchiolitis, including nebulizer and bronchodilator. However, the case group also received 5,000 units per kilogram of body weight of the injected vitamin A. The patients in both groups were visited every 24-hours during the hospitalization, at the time of discharge, and two weeks later, and the relevant information was recorded. The collected data was entered into the SPSS software version 24 and analyzed.

\section{Results}

In this study, 60 infants (25 (41.7\%) males vs. 35 (58.3\%) females) with a mean age of $7.63 \pm 4.08$ months were randomly divided into two equal groups $(\mathrm{n}=30)$ of case and control.

As Table 1 demonstrates, the mean age of infants did not show a statistically significant difference between the two groups $(\mathrm{P}>0.05)$.

As shown in Table 2, the prevalence of gender did not show a statistically significant difference between the two groups $(\mathrm{P}>0.05)$.

First: Determination and comparison of the severity of wheezing in infants with bronchiolitis at pre-treatment, 24 hours after treatment, during discharge, and two weeks after treatment in the two groups.As shown in Table 3, the severity of wheezing in infants before treatment and 24 hours after treatment did not show a statistically significant difference $(P>0.05)$. While $6.7 \%$ of infants in the control group had severe wheezing during discharge, no wheezing was reported in the case group. Also, the frequency of the moderate wheezing in the control and case groups was $40 \%$ and $10 \%$, respectively $(P=0.006)$. Also, two weeks after the treatment, the frequency of wheezing in control group was $30 \%$, but no wheezing was reported in case group, indicating a statistically significant difference $(\mathrm{P}<0.001)$.

Second: Determination and comparison of the status of retraction in infants with bronchiolitis at pre-treatment, 24 hours after treatment, during discharge, and two weeks after treatment in the two groups.

As shown in Table 4, the frequency of retraction severity in infants did not show a statistically significant difference between the two groups ( $\mathrm{P}>0.05)$.

Third aim: Determination and comparison of respiratory rate in infants with bronchiolitis at pre-treatment, 24 hours after treatment, during discharge, and two weeks after treatment in the two groups.As shown in the Table 5, the respiratory rate decreased significantly in both groups, and this difference existed until the end of the follow-up of patients due to the difference in baseline characteristics. 


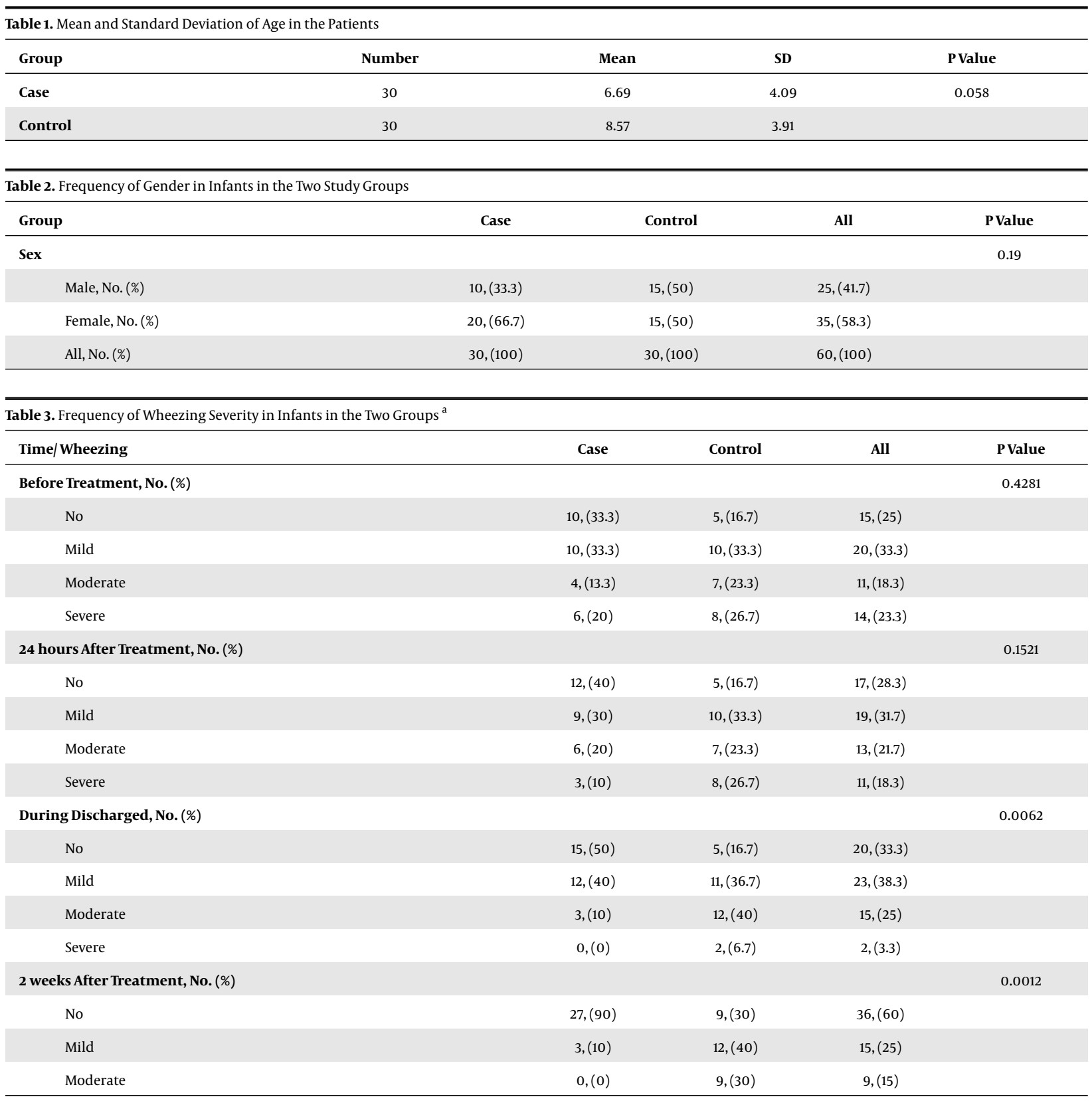

${ }^{a}$, Pearson Chi-square test; 2, Fisher's exact test.

Forth aim: Determining and comparing the general conditions of the infants with bronchiolitis at pretreatment, 24 hours after treatment, during discharge, and two weeks after treatment in the two groups. As shown in Table 6, general conditions did not show a statistically significant difference between the two groups $(\mathrm{P}>0.05)$.

\section{Discussion}

Bronchiolitis is a lower respiratory viral disease and a cause of wheezing (13). Diagnosis is generally based on history and clinical examination, and about $3 \%$ of infants are admitted due to bronchiolitis in the first year of infancy (6). Proper treatment and wheezing control can reduce the risk factors for asthma in infants and consequently reduce morbidity $(5,10,11)$. So far, no definitive treatment has been introduced for bronchiolitis, but the common treatments 


\begin{tabular}{|c|c|c|c|c|}
\hline Time/ Wheezing & Case & Control & All & P Value \\
\hline Before Treatment, No. (\%) & & & & 0.1741 \\
\hline No & $7,(23.3)$ & $13,(43.3)$ & $20,(33.3)$ & \\
\hline Intercostal & $13,(43.3)$ & $13,(43.3)$ & $26,(43.3)$ & \\
\hline Subcostal & $5,(16.7)$ & $1,(3.3)$ & $6,(10)$ & \\
\hline Severe & $5,(16.7)$ & $3,(10)$ & $8,(13.3)$ & \\
\hline 24 hour After Treatment, No. (\%) & & & & 0.0541 \\
\hline No & $9,(30)$ & $13,(43.3)$ & $22,(36.7)$ & \\
\hline Intercostal & $11,(36.7)$ & $3,(10)$ & $14,(23.3)$ & \\
\hline Treacheosternal & $7,(23.3)$ & $6,(20)$ & $13,(21.7)$ & \\
\hline Severe & $3,(10)$ & $8,(26.7)$ & $11,(18.3)$ & \\
\hline During Discharged, No. (\%) & & & & 0.2732 \\
\hline No & 28, (93.3) & $24,(80)$ & $52,(86.7)$ & \\
\hline Intercostal & $2,(6.7)$ & $5,(16.7)$ & $7,(11.7)$ & \\
\hline Treacheosternal & $0,(0)$ & $1,(3.3)$ & $1,(1.7)$ & \\
\hline 2 week After Treatment, No. (\%) & & & & 0.3552 \\
\hline No & $30,(100)$ & $28,(93.3)$ & $58,(96.7)$ & \\
\hline Intercostal & $0,(0)$ & $1,(3.3)$ & $1,(1.7)$ & \\
\hline Treacheosternal & $0,(0)$ & $1,(3.3)$ & $1,(1.7)$ & \\
\hline \multicolumn{5}{|c|}{ a1, Pearson Chi-square test; 2, Fisher's exact test. } \\
\hline Time & Number & Mean & SD & PValue \\
\hline Before Treatment & & & & 0.0091 \\
\hline Case & 30 & 46.86 & 9.86 & \\
\hline Control & 30 & 53.03 & 7.77 & \\
\hline 24 hours After Treatment & & & & 0.0011 \\
\hline Case & 30 & 45.63 & 9.25 & \\
\hline Control & 30 & 53.33 & 7.26 & \\
\hline During Discharged & & & & 0.0012 \\
\hline Case & 30 & 41.43 & 6.05 & \\
\hline Control & 30 & 47.06 & 4.98 & \\
\hline 2 weeks After Treatment & & & & 0.0192 \\
\hline Case & 30 & 40.03 & 7.36 & \\
\hline Control & 30 & 43.13 & 4.62 & \\
\hline
\end{tabular}

${ }^{a}$ 1, Pearson Chi-square test; 2, Fisher's exact test.

include using supportive cold nebulizer and bronchodilator (15).

Due to the role of vitamin A in the epithelium stabilization of the lower respiratory tract and immune system, (12) the present study aimed to investigate the effect of vitamin
A on persistent wheezing in 60 infants with bronchiolitis referred to Ali Ibn Abi Talib Hospital of Zahedan, Iran in 2018. The mean age and frequency of sex were not significantly different in the two groups.

The results of our study showed that the severity of 


\begin{tabular}{|c|c|c|c|c|c|c|}
\hline Time & General Conditions & Group & Case & Control & All & P Value \\
\hline \multirow[t]{6}{*}{ Before Treatment } & Normal & Case & 6 & 1 & 7 & \multirow{6}{*}{0.124} \\
\hline & & Control & 20 & 3.3 & 11.7 & \\
\hline & Difficult feeding & Case & 18 & 23 & 41 & \\
\hline & & Control & 60 & 76.7 & 68.2 & \\
\hline & Agitation & Case & 6 & 6 & 12 & \\
\hline & & Control & 20 & 20 & 20 & \\
\hline \multirow[t]{4}{*}{24 hours After Treatment } & Normal & Case & 26 & 20 & 46 & \multirow{4}{*}{0.125} \\
\hline & & Control & 86.7 & 66.7 & 76.7 & \\
\hline & Difficult feeding & Case & 4 & 10 & 14 & \\
\hline & & Control & 13.3 & 33.3 & 23.3 & \\
\hline
\end{tabular}

wheezing was not significantly different in infants before and 24 hours after treatment. However, while $6.7 \%$ of infants in the control group had severe wheezing during discharge, no wheezing was reported in the case group. Also, there was no significant difference between the severity of retraction and general conditions in the infants of the two groups. There was also a significant decrease in the respiratory rate in both groups, which was observed until the end of the follow-up.

Pinnock et al. evaluated children's respiratory diseases and reported that children with frequent episodes of respiratory problems benefited from vitamin A supplements. In a randomized clinical trial in 1988, they also investigated 206 children aged 2 to 7 years with a history of hospitalization during infancy due to bronchiolitis and treated them with vitamin A supplementation. The results showed that this supplement did not alter plasma retinol levels and did not affect respiratory morbidity (22). These results are in line with the findings of our study.

In our study, it was found that vitamin A supplementation in infants could improve wheezing, and it did not have a negative effect on the general conditions and retraction of patients.

Another study conducted by Dowell et al. (1996) in Chile aimed at assessing the effect of high-dose vitamin A on RSV infection in 180 children aged one month to six years old. Based on the specimen from the nose, the patients were treated with oral vitamin A(50,000 to 200,000 IU retinol palmitate based on age) and placebo. The results showed that there was no significant relationship between the administration of vitamin A and the reduction in hospitalization period, the need for oxygen, or the time of loss of hypoxemia. However, in the analysis of subgroups, it was found that the administration of this vitamin resulted in a significant reduction in the duration of tachyp- nea and shortening of the hospitalization period for children who had severe hypoxemia during admission (23). In our study, the duration of hospitalization was not studied, but it was found that administration of vitamin A could reduce wheezing; but its effect on reducing the respiratory rate was similar to that of the control group.

On the other hand, some studies have found that the prevalence of vitamin A deficiency is higher in patients with persistent wheezing, and serum retinol levels are associated with the severity of this wheezing (24). These results are in contrast with the results of our study. In our study, it was found that the administration of vitamin A in infants improved their wheezing and had no negative effects on the general conditions and retraction of patients.

Although the serum vitamin A level was not measured in our study, it could be concluded that the serum vitamin A level increased in the case group after treatment, which was associated with a decrease in the severity of wheezing. The reason for this difference may be due to the difference in the sample size, study design, demographic characteristics of the patients, and the effects of the confounders. Therefore, serum vitamin A levels are important at prior to treatment. (25).

In this regard, Sharafian et al. (2015) examined the effect of vitamin A on persistent wheezing after bronchiolitis in 84 one- to twelve-year-old infants in Yasouj, Iran. In this study, patients diagnosed with viral bronchiolitis were randomly assigned to two groups (case and control). The control group received conventional treatments for bronchiolitis, including cold nebulizer and wet oxygen with bronchodilator, and the case group received a vitamin A dose of 5000 units per kilogram in addition to the abovementioned treatment. The severity and rate of wheezing were examined in the two groups at pre-treatment, after the treatment, as well as one and two weeks after the treat- 
ment. After two weeks, there was a significant decrease in the severity and rate of wheezing in the group receiving vitamin A compared to the control group $(\mathrm{P}<0.05)$. The authors concluded that the administration of a dose of vitamin A in hospitalized patients due to viral bronchiolitis can significantly reduce post-bronchial wheezing (24). The results of this study are in line with the findings of our study.

Tian M et al. (2009) examined the causes of bronchiolitis in infants and reported that (a) lack of breastfeeding, exposure to cigarette smoke, and vitamin A and D deficiency were a major contributor to the onset of RSV bronchiolitis; (b) exposure to cigarette smoke, vitamin D deficiency, a history of atopy and a family history of atopy were important risk factors for wheezing after bronchiolitis in children; (c) patients with eosinophilia, high serum IgE, RANTES, and a decrease in the ratio of $\mathrm{TH} 1$ to $\mathrm{TH} 2$ increased the risk of wheezing (26). However, in our study, it was found that vitamin A has a direct relationship with the wheezing of patients, which is inconsistent with the results of the study. The reason for this difference may be due to the difference in sample size, type of study, demographic characteristics of patients, and the inclusion criteria. $T$

In a study by Ellison et al., vitamin A supplementation was associated with a $60 \%$ reduction in mortality in measles patients, which researchers attributed to the effect of vitamin A on the immune system and epithelium (27).

Pres-Chi et al., in a study in Santiago on admitted infants by diagnosis of RSV pneumonia showed that receiving vitamin A did not have a role in the duration of hospitalization, but it improved faster tachypnea in patients with oxygen levels lower than $90 \%$. They attributed the cause to the role of vitamin A in reducing inflammation of the lower respiratory tract, or its role in regulating the immune system (28).

In a study on serum vitamin A levels in 400 patients aged 3 to 36 months with wheezing, Shalaby et al. concluded that low levels of vitamin A are associated with persistent wheezing and acute respiratory disease (29). Although these results were in line with our findings, the study design was different from ours.

In a study in Australia on 206 children aged 2-7 years with a history of bronchiolitis leading to hospitalization, measuring serum levels of vitamin A and its administration showed that administration of vitamin A supplements could not raise serum retinol levels, and it did not affect the morbidity rate (30). These results are in contrast with the results of our study.

\subsection{Conclusion}

The results of this study showed that the severity of wheezing in infants receiving vitamin A significantly decreased during discharge and two weeks after treatment. Therefore, considering the prevalence of bronchiolitis in children and the high incidence of wheezing in infants following bronchiolitis, and given the direct effect of vitamin A on reducing infant wheezing, it is recommended that vitamin A along with other treatments be administrated for infants with symptoms of bronchiolitis.

\section{Acknowledgments}

The authors would like to thank the Faculty of Medicine of Zahedan University of Medical Sciences for supporting this study. This study was approved by the Ethics Committee of Zahedan University of Medical Sciences. This article has been taken from a doctoral thesis.

\section{Footnotes}

Authors' Contribution: Afsaneh Mirshekari conceived and designed the evaluation and drafted the manuscript. Gholamreza Soleimani participated in designing the evaluation and helped to draft the manuscript. Elham Shafighi Shahri re-evaluated the clinical data and revised the manuscript. Alireza Ganjali interpreted the clinical data and revised the manuscript. Alireza Ansari Moghadam interpreted the clinical data and revised the manuscript. All authors read and approved the final manuscript.

\section{Clinical Trial Registration Code: IRCT20180425039418N5.}

Conflict of Interests: The authors have no financial interest in the contents of the manuscript.

Ethical Approval: IR.ZAUMS.REC.1396.14.

Funding/Support: This study was supported by Zahedan University of Medical Sciences.

Informed Consent: An informed consent was obtained from the parents of all patients.

\section{References}

1. Robledo-Aceves M, Moreno-Peregrina MJ, Velarde-Rivera F, AscencioEsparza E, Preciado-Figueroa FM, Caniza MA, et al. Risk factors for severe bronchiolitis caused by respiratory virus infections among Mexican children in an emergency department. Medicine (Baltimore). 2018;97(9). eng. e0057. doi: 10.1097/md.0000000000010057. [PubMed: 29489664]. [PubMed Central: PMC5851717]. 
2. Cui D, Feng L, Chen Y, Lai S, Zhang Z, Yu F, et al. Clinical and Epidemiologic Characteristics of Hospitalized Patients with LaboratoryConfirmed Respiratory Syncytial Virus Infection in Eastern China between 2009 and 2013: A Retrospective Study. PLoS One. 2016;11(11). eng. e0165437. doi: 10.1371/journal.pone.0165437.[PubMed: 27802292]. [PubMed Central: PMC5089734].

3. Øymar K, Skjerven HO, Mikalsen IB. Acute bronchiolitis in infants, a review. Scand J Trauma Resusc Emerg Med. 2014;22:23. eng. doi: 10.1186/1757-7241-22-23. [PubMed: 24694087]. [PubMed Central: PMC4230018].

4. Soleimani G, Akbarpour M. Clinical presentation of novel influenza a $(\mathrm{h}(1) \mathrm{n}(1))$ in hospitalized children. Iran J Pediatr. 2011;21(2):215-9. eng. [PubMed: 23056790]. [PubMed Central: PMC3446154].

5. Mansbach JM, Luna PN, Shaw CA, Hasegawa K, Petrosino JF, Piedra $\mathrm{PA}$, et al. Increased Moraxella and Streptococcus species abundance after severe bronchiolitis is associated with recurrent wheezing. J Allergy Clin Immunol. 2020;145(2):518-52700000000. eng. doi: 10.1016/j.jaci.2019.10.034. [PubMed: 31738994]. [PubMed Central: PMC7010548].

6. Nair H, Nokes DJ, Gessner BD, Dherani M, Madhi SA, Singleton RJ, et al. Global burden of acute lower respiratory infections due to respiratory syncytial virus in young children: a systematic review and meta-analysis. Lancet. 2010;375(9725):1545-55. eng. doi: 10.1016/s0140-6736(10)60206-1. [PubMed: 20399493]. [PubMed Central: PMC2864404].

7. Živić H. P658 Association of severe viral bronchiolitis during infancy and early life with development of recurrent wheeze. BMJ Publishing Group Ltd; 2019.

8. Franklin D, Babl FE, Schlapbach LJ, Oakley E, Craig S, Neutze J, et al. A Randomized Trial of High-Flow Oxygen Therapy in Infants with Bronchiolitis. N Engl J Med. 2018;378(12):1121-31. eng. doi: 10.1056/NEJMoa1714855. [PubMed: 29562151].

9. Marcdante K, RM K. Nelson essentials of pediatrics 7th ed. Philadelphia: Saunders Elsevier; 2015.

10. Soleimani G, Shafighi Shahri E, Rashidi S, Salari Z, Ansari Moghadam A. Epidemiology, clinical, and laboratory characteristics of bronchiolitis in hospitalized children.J Compr Ped. 2014;5(3).

11. Chen S, Gu W, Wu M, Hao C, Zhu C, Shao X, et al. Risk factors for recurrent wheezing after bronchiolitis in infants: 2-year follow up in China. BMC Infect Dis. 2021;21(1):250. eng. doi:10.1186/s12879-021-059378. [PubMed: 33691633]. [PubMed Central: PMC7945297].

12. Rinawi F, Kassis I, Tamir R, Kugelman A, Srugo I, Miron D. Bronchiolitis in young infants: is it a risk factor for recurrent wheezing in childhood? World JPediatr. 2017;13(1):41-8. eng. doi: 10.1007/s12519-0160056-4. [PubMed: 27650524].

13. Fretzayas A, Moustaki M. Etiology and clinical features of viral bronchiolitis in infancy. World J Pediatr. 2017;13(4):293-9. eng. doi: 10.1007/s12519-017-0031-8. [PubMed: 28470580]. [PubMed Central: PMC7090852].

14. Stephensen $C B$, Lietz $G$. Vitamin A in resistance to and recovery from infection: relevance to SARS-CoV2. Br J Nutr. 2021;126(11):1663-72. eng. doi: 10.1017/s0007114521000246. [PubMed: 33468263]. [PubMed Central: PMC7884725].

15. Arikan S, Durusoy C, Akalin N, Haberal A, Seckin D. Oxidant/antioxidant status in recurrent aphthous stomatitis. Oral Dis.
2009;15(7):512-5. eng. doi: 10.1111/j.1601-0825.2009.01580.x. [PubMed: 19761497].

16. Johnson EJ, Russell RM. Beta-Carotene. In: Coates PM, Betz JM, Blackman MR, Cragg GM, Levine M, Moss J, et al., editors. Encyclopedia of dietary supplements. 2nd ed ed. London and New York: Informa Healthcare; 2010.

17. Ross CA. Vitamin A. In: Coates PM, Betz JM, Blackman MR, editors. Encyclopedia of dietary supplements. 2nd ed ed. London and New York: Informa Healthcare; 2010. p. 778-91.

18. Ross A. Vitamin A. In: Shils M, Shike M, Ross A, Caballero B, Cousins R, editors. Modern Nutrition in Health and Disease. 10th ed ed. Baltimore, MD: Lippincott Williams \& Wilkins; 2006. p. 351-75.

19. Solomons NW. Vitamin A. In: Bowman B, Russell R, editors. Present knowledge in nutrition. 9th ed ed. Washington, DC: International Life Sciences Institute; 2006. p. 157-83.

20. Ali AS, Abolghasem J; et al. study of iron, folic acid, B12 and vitamin A of umbilical cord blood in relation to weight and height of birth. Res Med.2000;26(1):37-41.

21. Villamor E, Fawzi WW. Effects of vitamin a supplementation on immune responses and correlation with clinical outcomes. Clin Microbiol Rev. 2005;18(3):446-64. eng. doi: 10.1128/cmr.18.3.446-464.2005. [PubMed: 16020684]. [PubMed Central: PMC1195969].

22. Pinnock CB, Douglas RM, Martin AJ, Badcock NR. Vitamin A status of children with a history of respiratory syncytial virus infection in infancy. Aust Paediatr J. 1988;24(5):286-9. eng. doi: 10.1111/j.14401754.1988.tb01364.x. [PubMed: 3067695].

23. Dowell SF, Papic Z, Bresee JS, Larrañaga C, Mendez M, Sowell AL, et al. Treatment of respiratory syncytial virus infection with vitamin A: a randomized, placebo-controlled trial in Santiago, Chile. Pediatr Infect Dis J. 1996;15(9):782-6. eng. doi: 10.1097/00006454-19960900000009. [PubMed: 8878221].

24. Sharafian S, Keshavarz K, Abasi R, Zoladl M, Asadi S H, Kazeroni S, et al. The Effect (s) of Vitamin A on Persistent Wheezing after Bronchiolitis in Infants Aged 1-12 Months. Armaghane Danesh. 2015;20(1):1-10.

25. Keshavarz K, Ardekani SS, Zoladl M, Rezakhani A, Abasi R. PO-1018 The Role Of Vitamin A In Post Bronchiolitis Wheezing In Infants 2 To 12 Months Old. BMJ Publishing Group Ltd; 2014.

26. Tian M, Zhao D, Wen G, Shi S. The correlation factor about respiratory syncytial virus bronchiolitis and post-bronchiolitis wheezing in infant. Zhonghua Shi Yan He Lin Chuang Bing Du Xue Za Zhi. 2009;23(5):371-4.

27. Ellison JB. INTENSIVE VITAMIN THERAPY IN MEASLES. $\mathrm{Br}$ Med J. 1932;2(3745):708-11. eng. doi: 10.1136/bmj.2.3745.708. [PubMed: 20777111]. [PubMed Central: PMC2521770].

28. Caballero MT, Polack FP, Stein RT. Viral bronchiolitis in young infants: new perspectives for management and treatment. J Pediatr (Rio J). 2017;93 Suppl 1:75-83. eng. doi: 10.1016/j.jped.2017.07.003. [PubMed: 28859915].

29. Shalaby SA,Al-Harbi KM, El-Tarhouny SA. Vitamin A status in wheezing Saudi children. Saudi Med J. 2011;32(5):510-4. eng. [PubMed: 21556473].

30. Homaira N, Wiles LK, Gardner C, Molloy CJ, Arnolda G, Ting HP, et al. Assessing the quality of health care in the management of bronchiolitis in Australian children: a population-based sample survey. BMJQual Saf. 2019;28(10):817-25. eng. doi: 10.1136/bmjqs-2018-009028. [PubMed: 30940731]. [PubMed Central: PMC6837255]. 